EPJ Web of Conferences 110,01053 (2016)

DOI: $10.1051 /$ epjconf/201611001053

(C) Owned by the authors, published by EDP Sciences, 2016

\title{
THERMOPHYSICAL REACTIVITY CONTROL OF RBMK-1000
}

\author{
Aleksander V. Vorobiev ${ }^{1, \text { a }}$, Aleksandra M. Antonova ${ }^{2}$ and Maksim P. Vinogradov ${ }^{3}$ \\ ${ }^{1}$ National Research Tomsk Polytechnic University, 634050 Tomsk, Russia
}

\begin{abstract}
In this paper, viewed thermophysical characteristics of the moderator water graphite reactor RBMK. Indicated possibilities of controlling thermal state of graphite stack by regulation composition of the purge gas. Presents experimental results, but static thermal state characteristics of graphite moderator RBMK-1000. Developed a software code for integral characteristic engineering calculations, that determine value of margin reactivity reactor RBMK-1000, in the slow transients.
\end{abstract}

\section{Introduction}

Essential factor that determine the reliability and safety of nuclear power plants with water-graphite reactors, is the reliability of the graphite moderator (stack).

RBMK-1000 graphite stack has cylindrical form (diameter $11,8 \mathrm{~m}$ and height $7 \mathrm{~m}$ ), which is composed of 2488 separate columns with cross section $0,25 * 0,25 \mathrm{~m}$. In the axial bore of those columns are installed process tubes (PC, CPS etc.). The fuel assemblies, which is installed inside the process channels, are cooled by water with a temperature difference $250 \ldots 280{ }^{\circ} \mathrm{C}$.

While reactor in operation, part of $(5 \ldots 6 \%)$ reactor thermal output is releasing in graphite stack. This heat is transferred to process channels. To ensure thermal contact with carbon blocks there are carbon packing rings on the process tubes and the purge gas is supplied into the gaps between block and PC (the standard composition is $10 \%$ nitrogen and $90 \%$ helium by volume).

RBMK units design allows changing the composition of the purge mixture [1].

Graphite stack temperature is primarily depends on thermal reactor output and nitrogen concentration in purge mixture (further $\left.C_{N}\right)$, because its components have different thermal conductivity coefficients.

In practice, it is assumed that in all reactor operation regimes maximum graphite temperature must not exceed the threshold temperature of $750 \ldots 800{ }^{\circ} \mathrm{C}$ [1], in which the oxidation of graphite in the presence of water vapor is more intense. Essential factor that determine the reliability and safety of nuclear power plants with water-graphite reactors, is the reliability of the graphite moderator (stack), especially in transient regimes. Changes in reactor power associated with thermal cycling stress in graphite and can lead to premature failure of graphite stack [2,3]. Moreover, fluctuations in the average graphite stack (moderator) temperature lead to corresponding changes in the reactor reactivity, because the RBMK units have a significant positive moderator temperature coefficient on reactivity feedback effect $[1,4,5]$.

The dependence between change in temperature of graphite and the composition of purge mixture can be used to improve the efficiency of the RBMK reactors at fractional loads. For example, while reducing reactor power, increasing the nitrogen content $C_{N}$ in purge mixture leads to degradation of heat transfer from the graphite, thus increase its temperature, in such a way temperature and reactivity are compensated due to changes in the power [5].

Therefore, the crucial task is to determine temperature characteristics of RBMK graphite stack and substantiation ways to control its thermal state.

\section{Model development}

The overarching goal is thermal resistance $R_{t h}$ calculation in a gap between block and process tube. The problem of evaluation dependence $R_{t h}$ on basic parameters involves steady heat conduction in axial-symmetric area that includes a part of the graphite block, two half rings of solid contact, gas gaps. The calculations were made by MPEI method [6].

\footnotetext{
${ }^{a}$ Corresponding author: worob@tpu.ru
} 


\section{EPJ Web of Conferences}

Lindsay-Bromley equation for purge mixture thermal conductivity $\lambda_{\text {mix }}$ has been chosen among a large number because of satisfactorily (maximum error 7.5\%) describing the experimental data [5]. Y.P. Shlykov method was used to determine thermal contact resistance [7].

The resistance calculation complexity $R_{t h}$ force us to get the multifactorial description of gap between block and process tube, and chose predetermined range of planing experiments as base of research method.

The objective function is a value $R_{t h}$. Essential factors and their variability intervals are selected according to process channel mean thermal characteristics: $q_{l . g r}=(0,25 \ldots 4,25) \cdot 104 \mathrm{~W} / \mathrm{m} ; \lambda_{g r}=10 \ldots 40 \mathrm{~W} /\left(\mathrm{m} \cdot{ }^{\circ} \mathrm{C}\right) ; x_{N}=0 \ldots 1 ; \delta_{g r}=0,5 \ldots 1,5 \mathrm{~mm} ; p_{k n}=0 \ldots 0,5 \mathrm{MPa}$. Geometric shape choice and evaluation approximate equations parameters were performed with a least square method (LSM).

In a general way, resulted regression equation form is

$$
R_{t h}=a_{0}+a_{1} \cdot \lambda_{g r}+a_{2} \cdot p_{\kappa H}+a_{3} \cdot \delta_{2}+a_{4} \cdot x_{N}+a_{5} \cdot q_{l . g r}+a_{6} \cdot \lambda_{g r}^{2}+a_{7} \cdot \lambda_{g r} \cdot p_{k n}+\ldots,
$$

where, $a_{0}, a_{1}, a_{2} \ldots$ - regression coefficients.

The nonsignificant terms with (Student's t-test, probability belief $\alpha=0.9$ ) regression coefficients were neglected. Approximation errors do not exceed $5 \%$ due to equation (3). Analysis shows that the influence coefficient $k_{C}$ varies from 0,7 to $1,3{ }^{\circ} \mathrm{C} / \%$ and coefficient $k_{Q}$ - from 0.07 to $0.11^{\circ} \mathrm{C} / \mathrm{MW}$, accordingly to the governing parameters range.

Experimental results which were performed at the first RBMK-1000 unit Leningrad NPP, confirm analytic data.

\section{Field research}

To measure the main reactor parameters were used standard equipment and a centralized control system, because of heavy NPP equipment operation conditions.

Purge mixture composition was changing in steps at different levels of the reactor thermal power to determine the static characteristics of graphite stack.

As the output value was considered the average temperature $t_{\text {g.col }}$ of the masonry joints graphite columns.

After the initial result processing, only eighteen experiments have statistically significant contribution (Fig. 1). The linear and square polynomials were selected to approximate them and estimation of polynomial parameters was made by LSM [8]. F-test was used to check adequacy of models; significance of (level $\alpha=0,9)$ regression coefficients - Student's test.

$$
\begin{aligned}
& t_{g . c o l}=b_{0}+b_{1} \cdot Q_{n r}+b_{2} \cdot C_{N}+b_{3} \cdot Q_{n r}^{2}+b_{4} \cdot Q_{n r} \cdot C_{N}+b_{5} \cdot C_{N}^{2}, \\
& t_{g . c o l}=d_{0}+d_{1} \cdot Q_{n r}+d_{2} \cdot C_{N},
\end{aligned}
$$

where, $\quad b_{0}, b_{1}, b_{2}, \ldots, d_{0}, d_{1}, \ldots-$ numerical values of regression coefficients.

Parameters evaluation of square polynomial (3) allows determine the value of integral coefficients influence of reactor thermal power $k_{Q}$ and purge mixture composition $k_{c}$ on graphite moderator temperature $t_{\text {g.col. }}$.

Coefficient $k_{Q}$ heavily depends on the composition of mixture in stack: changing mixture composition from helium to nitrogen leads to increase in $k_{Q}$ more than 2 times $\left(0.0643\right.$ to $\left.0,1264{ }^{\circ} \mathrm{C} / \mathrm{MW}\right)$. The coefficient $k_{c}$ depends on reactor thermal output: the change $Q_{n r}$ from 3200 to $1600 \mathrm{MW}$ ratio $k_{c}$ decreases from 1.98 to $0,99{ }^{\circ} \mathrm{C} / \%$. 


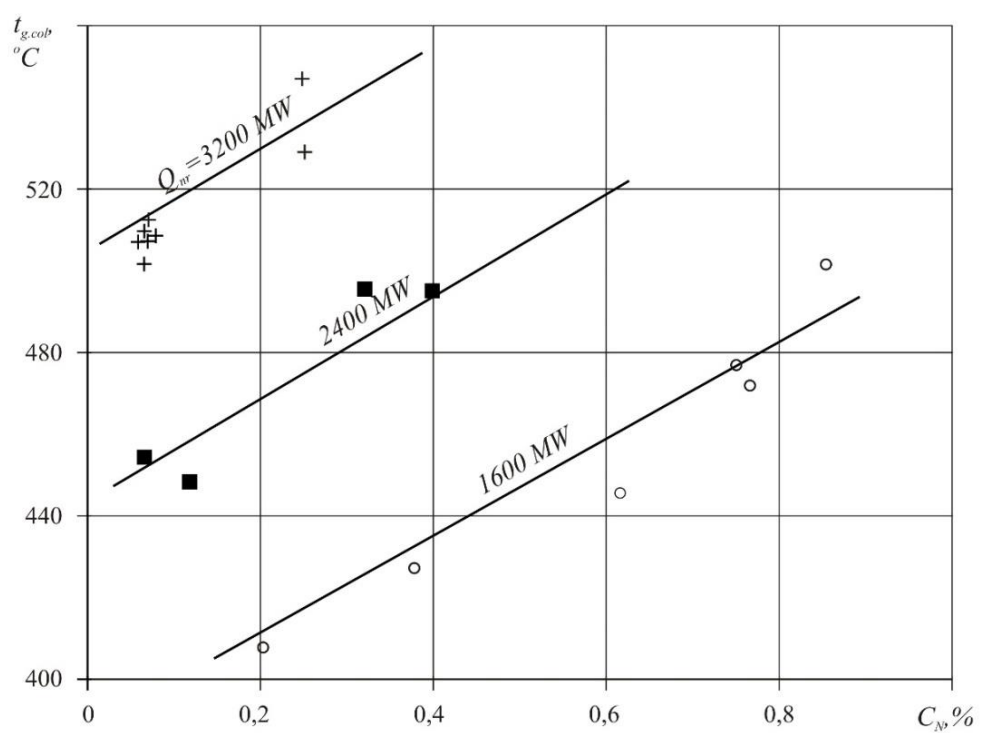

Figure 1. Change in RBMK-1000 moderator average temperature with composition of purge mixture and reactor thermal output: --- - evaluation by equation $(3) ;+, \mathbf{m}, \circ-$ experimental data at different loads.

\section{Calculation research}

The object-oriented visual programming Delphi was used to solve the problem.

The modeling code designed to calculate the relatively slow transient processes in the RBMK-1000 reactor. The algorithm, which is implemented in the program code, includes hydraulic processes in the reactor core and, in particular, graphite moderator and also transient xenon poisoning is regarded in point approximation.

In equations describing the dynamics of graphite temperature used numerical values of constants (coefficients of influence $k c, k Q$ etc.), which were determined in computational research and field experiments at RBMK-1000 reactors.

To check the model, graphite moderator thermal state test calculations have been carried out while varying thermal power $Q_{n r}$ and composition $C_{N}$ of purge gas. The results of one of these calculations and the experimental points are shown in Fig. 2. 


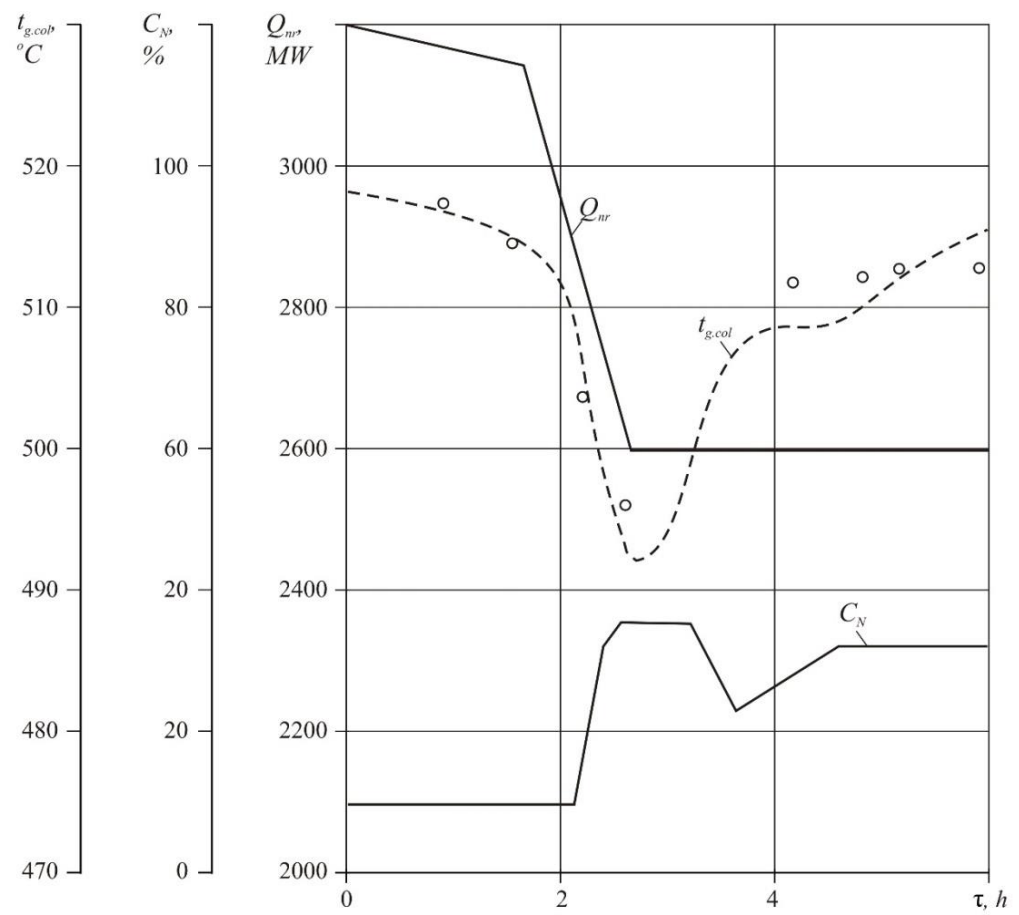

Figure 2. Change RBMK-1000 parameters with time: ----- and - - model simulation; $\circ$ - experimental data.

Fig. 2 analysis indicates a satisfactory agreement between the calculated data by model with experimental data points and possibility its further use in computational research.

\section{Conclusion}

After calculation research were defined static factors influence kQ power unit and the composition of the purge gas at a temperature moderator $\mathrm{kC}$ water-graphite reactor $\mathrm{RBMK}-1000$. In the investigated range of parameters determining factor influence coefficient $\mathrm{kC}$ varies from 0,7 to $1,3^{\circ} \mathrm{C} / \%$ and coefficient $\mathrm{kQ}$ - from 0.07 to $0.11^{\circ} \mathrm{C} / \mathrm{MW}$, accordingly to the governing parameters range.

The results of RBMK-1000 reactors field experiments generally confirmed the calculation research. changing mixture composition from helium to nitrogen leads to increase in kQ more than 2 times $\left(0.0643\right.$ to $\left.0,1264{ }^{\circ} \mathrm{C} / \mathrm{MW}\right)$, and changing thermal output from 3200 to $1600 \mathrm{MW}$ ratio $\mathrm{kC}$ decreases from 1.98 to $0,99{ }^{\circ} \mathrm{C} / \%$.

As the result, a software code was developed for integral characteristic engineering calculations, that determine value of margin reactivity reactor RBMK-1000, in the slow transients.

\section{References}

1. N.A. Dollezhal, Duct nuclear power reactors (M .: Atomizdat, 1980)

2. A.V. Golovatsky, V.N. Nesterov, I.V. Shamanin, Bulletin of the Tomsk Polytechnic University, 319 (2), 71 (2011)

3. A.A. Tsyganov, Bulletin of the Tomsk Polytechnic University, 312 (2), 32 (2008)

4. B.C. Romanenko, Ser. Physics and technology of nuclear reactors, 5 (18), 8 (1981)

5. A.V. Vorobiev, Office of the composition of the gas mixture blown through the graphite stack reactions RBMK-torus, to improve the reliability and efficiency of his work, 20 (1988)

6. B.A. Dementiev, Nuclear power reactors ( M .: Energoatoamizdat, 352, 1984)

7. Y.P. Shlikov, Contact thermal resistance (M .: Energia, 328, 1977)

8. A.A. Borovkov, Math statistics. Parameter estimation, hypothesis testing ( Nauka, 312,1984) 